\title{
The effect of a decision-support mHealth application on maternal and neonatal outcomes in two district hospitals in Rwanda: pre - post intervention study
}

\author{
Aurore Nishimwe ${ }^{1,2^{*}} \mathbb{0}$, Latifat Ibisomi ${ }^{1,3}$, Marc Nyssen $^{4}$ and Daphney Nozizwe Conco ${ }^{1}$
}

\begin{abstract}
Background: Globally, mobile health (mHealth) applications are known for their potential to improve healthcare providers' access to relevant and reliable health information. Besides, electronic decision support tools, such as the Safe Delivery mHealth Application (SDA), may help to reduce clinical errors and to ensure quality care at the point of service delivery. The current study investigated the use of the SDA and its relationship to basic emergency obstetric and newborn care (BEmONC) outcomes for the most frequent complications in Rwanda; post-partum haemorrhage $(\mathrm{PPH})$ and newborn asphyxia.
\end{abstract}

Methods: The study adopted a pre-post intervention design. A pre-intervention record review of BEmONC outcomes: Apgar score and PPH progressions, was conducted for 6 months' period (February 2019 - July 2019). The intervention took place in two district hospitals in Rwanda and entails the implementation of the SDA for 6 months (October 2019- March 2020), and included 54 nurses and midwives using the SDA to manage PPH and neonatal resuscitation. Six months' post-SDA intervention, the effect of the SDA on BEmONC outcomes was evaluated. The study included 327 participants (114 cases of PPH and 213 cases of neonatal complications). The analysis compared the outcome variables between the baseline and the endline data. Fisher's exact test was used to compare the proportions and test between-group differences and significance level set at $p<0.05$.

Results: Unstable newborn outcomes following neonatal resuscitation were recorded in $62 \%$ newborns cases at baseline and $28 \%$ newborns cases at endline, $P$-value $=0.000$. Unstable maternal outcomes following PPH management were recorded in 19\% maternal cases at baseline and $6 \%$ maternal cases at endline, $P$-value $=0.048$. There was a significant association between the SDA intervention and newborns' and maternal' outcomes following neonatal resuscitation and PPH management, 6 months after baseline.

Conclusion: The use of the SDA supported nurses and midwives in the management of PPH and neonatal resuscitation which may have contributed to improved maternal and neonatal outcomes during 6 months of the SDA intervention. The findings of this study are promising as they contribute to a broader knowledge about the effectiveness of SDA in low and middle income hospital settings.

\footnotetext{
*Correspondence: naurore@cartafrica.org

${ }^{1}$ School of Public Health, Faculty of Health Sciences, University

of the Witwatersrand, 1 Smuts Avenue, Braamfontein,

Johannesburg 2000, South Africa

Full list of author information is available at the end of the article
}

(c) The Author(s) 2022. Open Access This article is licensed under a Creative Commons Attribution 4.0 International License, which permits use, sharing, adaptation, distribution and reproduction in any medium or format, as long as you give appropriate credit to the original author(s) and the source, provide a link to the Creative Commons licence, and indicate if changes were made. The images or other third party material in this article are included in the article's Creative Commons licence, unless indicated otherwise in a credit line to the material. If material is not included in the article's Creative Commons licence and your intended use is not permitted by statutory regulation or exceeds the permitted use, you will need to obtain permission directly from the copyright holder. To view a copy of this licence, visit http://creativecommons.org/licenses/by/4.0/. The Creative Commons Public Domain Dedication waiver (http://creativeco mmons.org/publicdomain/zero/1.0/) applies to the data made available in this article, unless otherwise stated in a credit line to the data. 
Keywords: Maternal outcome, Neonatal outcome, BEmONC, mHealth, Safe delivery application, Rwanda

\section{Background}

Mhealth refers to the practice of medicine and public health, supported by mobile devices (mobile phones, smartphones, tablets, smartwatches) [1]. Evidence suggests that mHealth applications are innovative approaches to improve the delivery of health care services [2]. Further, mhealth tools can help to reduce clinical errors and ensure quality care at the point of service delivery. There is growing evidence of the effectiveness of mHealth interventions in maternal and newborn health programs in low and middle-income countries (LMICs), particularly to improve adherence to treatment, meeting appointments, facilitate data collection, and the development of supportive networks for health professionals [3-6].

Among the many interventions currently being implemented to support maternal and newborn healthcare services, mHealth applications have been widely used in low-resources settings $[4,7-9]$ as a potential solution to maximize health providers' efficiency, health outcomes [9-11] and improve service utilization [2]. Common areas of application of mHealth tools include point-ofcare decision-making support; provider-to-provider communication; and data collection [12, 13]. Though mHealth interventions are well received by healthcare providers $[6,14,15]$ information about their effectiveness with regards to patient outcomes is limited $[5,9,16]$.

Many interventions on the use of mHealth for improving maternal and newborns' health outcomes in subSaharan Africa mainly focused on timely access to health facilities including reminders for antenatal appointments and referrals of mothers [17]. For instance, the CommCare mHealth technology is a digital solution that has shown promising results in assisting community health workers (CHWs) in data collection, decision support, communications with clients and health centres, and access to educational training materials [18]. Few mHealth applications are addressing the support of healthcare providers at the point of care with the main focus on clinical decision support in order to improve maternal and newborns outcomes [5, 9, 11, 19-22]. The Safe Delivery mHealth Application (SDA) is one of the recent mHealth applications (loaded in smartphones) which avails BEmONC clinical guidelines to support nurses and midwives' clinical decisions [23].

Rwanda, a sub-Saharan African country, reports high maternal and neonatal deaths. In 2015, Rwanda's maternal mortality was estimated at 290 deaths per 100,000 live births and its neonatal mortality rate was 20 deaths per 1000 live births, respectively [24] despite 90\% of deliveries taking place in healthcare facilities attended by health professionals $[25,26]$. Common causes of these deaths are preventable including postpartum hemorrhage $(22.7 \%$ of all documented cases) and newborn asphyxia and its complications (38\%) [27-29]. Challenges faced by healthcare providers in Rwanda include poor access to clinical guidelines and the lack of timely response to pregnancy complications during delivery care $[30,31]$. It is in this context that a mHealth application - a clinical decision support tool (SDA) to facilitate easy access to maternal and neonatal guidelines for routine and emergency obstetric and neonatal care was introduced. The SDA has been tested for its effectiveness through a randomized controlled trial conducted in Ethiopia [32]. The SDA has also shown a positive effect on nurses and midwives' knowledge and skills in the management of $\mathrm{PPH}$ and neonatal resuscitation in Rwanda [33]. This study investigated the use of the SDA and its relationship to BEmONC outcomes for the most frequent birth-related complications of PPH and newborn asphyxia in Rwanda. The current paper addresses one objective of a broad study about implementation of the SDA in Rwanda.

\section{Methods}

\section{Study design}

This was a pre-post intervention study done over a 14-month period. The study was conducted in three phases. The first phase was the pre-SDA intervention baseline study over a period of 6 months (February 2019 - July 2019). During this phase, a record review was conducted to document maternal and neonatal outcomes, PPH and Apgar score progressions, at baseline. The second phase, the SDA intervention (August 2019 - September 2019) comprised of the capacity building of nurses and midwives on the usage of the SDA; the SDA provision; the SDA piloting, and the launch of the SDA which marked the start of the SDA implementation. More details on the SDA training are documented in the protocol paper for this study [34]. The last phase was the 6 months of post-SDA intervention (October 2019- March 2020). Phase three encompassed the implementation of the SDA and another record review was conducted to document maternal and neonatal outcomes, PPH and Apgar score progressions, at endline. The data were collected from the delivery registries using data extraction forms. The collected data included patients' characteristics and information on maternal and 
newborn outcomes, Apgar scores and PPH progressions, following NR and PPH management. The data were collected by the researcher and four research assistants. Data accuracy and transcription were checked by the researcher before analysis.

\section{Study setting}

The study was conducted in two district hospitals in Rwanda: Masaka hospital in Kigali, an urban province; and Nyamata hospital located in the eastern rural province [26]. The two hospitals were chosen out of 12 district hospitals in the two provinces because both recorded a high number of deliveries per year [24]. Compared to other rural-based district hospitals, Nyamata reported the highest maternal and neonatal mortality rates [30].

\section{Study participants}

The study participants included records on neonatal complication cases and PPH cases. The inclusion criteria for the cases of neonatal complications were set as babies born with asphyxia (Apgar score $\leq 7$ ) and were subject to neonatal resuscitation. We excluded preterm births ( $\leq 36$ completed weeks of gestation) and births with major congenital malformations because prematurity and congenital complications other than the newborn asphyxia could influence the newborn outcome after resuscitation [35]. We have also excluded records with missing data on Apgar scores and neonatal outcome. On the other hand, the inclusion criteria for PPH cases were set as women who had a recorded amount of blood loss more than or equal to $500 \mathrm{mls}$ and were subjected to PPH management. The records with missing data on PPH progression and maternal outcomes were also excluded. The study was conducted in three phases. Phase one, preintervention, included 126 cases of newborns asphyxia and 67 cases of PPH. Phase two, intervention, involved 33 midwives and 21 nurses who used the SDA in both Masaka and Nyamata district hospitals. Nurses and midwives were chosen because they are the frontline healthcare providers in childbirth care in district hospitals of Rwanda. The inclusion criteria for nurses and midwives were set as follows: having a work experience of at least 6 months in obstetric care and willing to participate in the study. All nurses and midwives $(n=54)$ working in the maternity departments of the selected hospitals volunteered to participate in the study and they were trained on the use of SDA. Phase three, postintervention, comprised 87 cases of newborns asphyxia and 47 cases of PPH. The flowchart in Fig. 1 shows the number of neonatal complication cases and PPH cases considered in the pre-intervention group (before the introduction of SDA) and the post-intervention group (after SDA).

\section{Description of the intervention: implementation of the safe delivery mhealth application (SDA)}

The SDA is a smartphone application developed by the Maternity Foundation, Copenhagen University, and the University of Southern Denmark. It is designed to support nurses and midwives in clinical decision making, by providing them with instant access to evidence-based BEmONC clinical guidelines and a selection of essential preventive protocols in a user-friendly format. The content of the SDA is primarily based on WHO (World health organization) clinical $\mathrm{BEmONC}$ guidelines and has been validated by an international group of global health experts [36]. The SDA contains easy to understand animated instruction videos, action cards with validated clinical guidelines, essential drugs lists with their indications, practical procedures guidelines, and a learning platform [32]. All features and functions in the SDA are designed for low-literacy and low-income settings and work offline once downloaded. The SDA can be used as a job aid and as an instructive aid in emergencies. While practicing, the skilled birth attendant (SBA) can consult action cards and drug lists to be helped in his/her clinical decision making. In the free time, the SBA can again play animated instruction videos, consult procedures descriptions and use the learning platform to update his/ her knowledge and skills. The SDA can be downloaded free of charge for iPhone at https://itunes.apple.com/dk/ app/safe-delivery/id985603707?mt $=8$ and for Android at https://play.google.com/store/apps/details?id=dk.mater nity.safedelivery\&hl=en.

The nurses and midwives in both hospitals received an explanation of the nature and purpose of the research and half-day training session on the use of the SDA. The majority of participants have downloaded the SDA on their personal smartphones during the training. The training session encompassed: an introduction to the research project; an overview of the SDA development and global outreach; description of the SDA features and modules; and hands-on practice on using the SDA as a job-aid and as a learning tool. Each of the study settings (Masaka and Nyamata) received three smartphones, with pre-installed English and French versions of the SDA. This was followed by the 6 months' intervention period, during which nurses and midwives provided BEmONC services with the assistance of the SDA installed on their personal smartphones or the smartphones provided by the researcher. During this period, the provided six smartphones were made available to the team on duty (three staffs per shift per hospital) at all times for use as a backup in case the participants experienced problems 


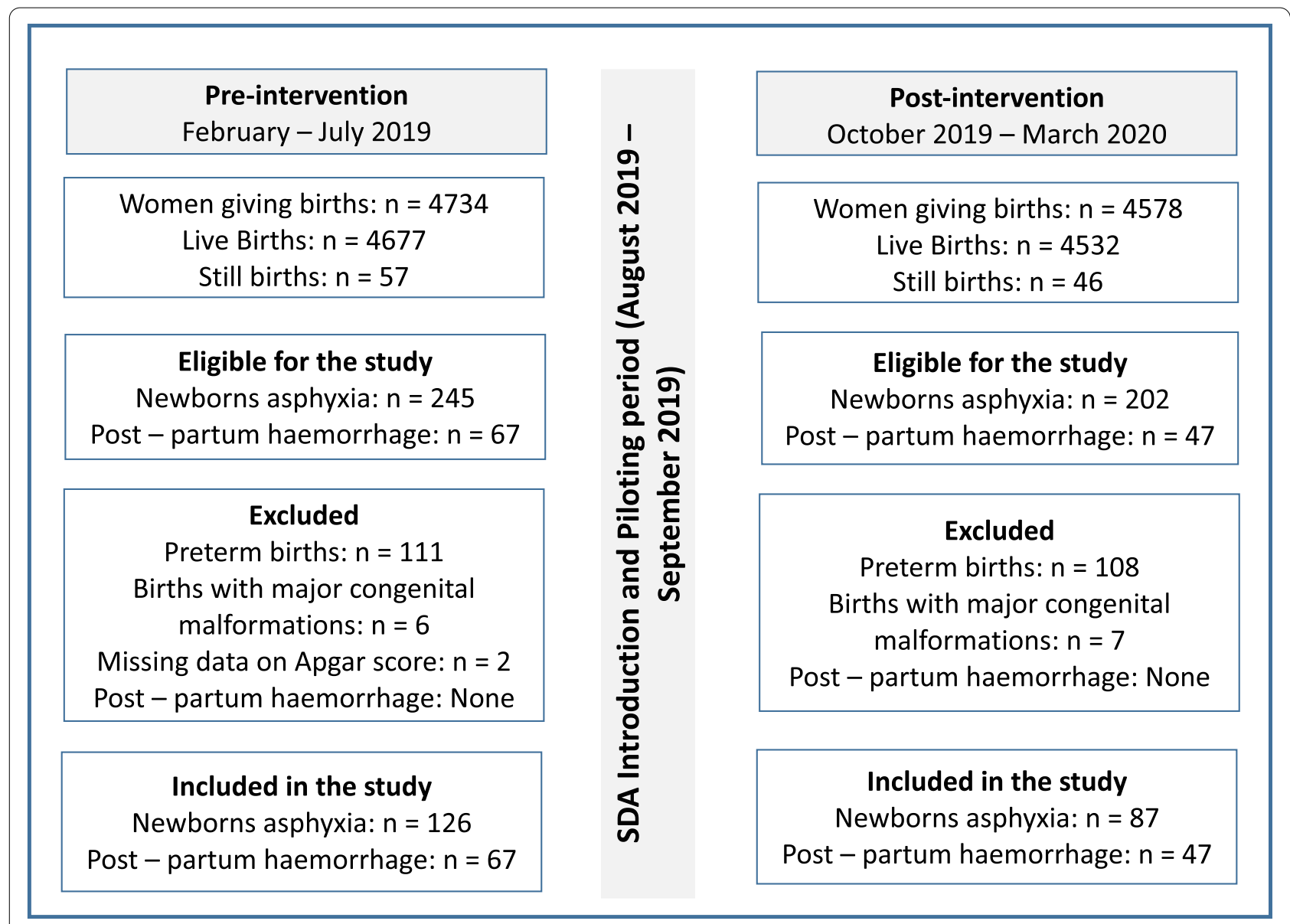

Fig. 1 Records review flow chart

with their own smartphones. Nurses and midwives were expected to use the SDA as often as they wished. The intervention also entailed two visits per week by two research assistants to each of the study hospitals. During the visits, the research assistants monitored the SDA use by nurses and midwives.

\section{Data collection}

Data extraction forms were used to retrieve information on all cases of PPH and neonatal complications recorded in delivery registers. These were extracted and reviewed 6 months' pre-SDA intervention and 6 months' post-SDA intervention. The delivery registers contain information on obstetric and neonatal care including the Apgar scores and $\mathrm{PPH}$ progressions following neonatal resuscitation and $\mathrm{PPH}$ management. Newborn deaths or admission to the neonatal intensive care unit (NICU) or to neonatologist's service due to an unstable outcome (Apgar score $<7$ ) after neonatal resuscitation was used as the primary outcome measure to determine the failure of neonatal resuscitation. The mothers' referral to a higher-level facility (referral hospital) following an unstable outcome (persistent bleeding) after PPH management was considered as the primary outcome to measure the failure of PPH management at the district hospital. On the newborn side, we obtained data on Apgar scores progression and neonatal outcome following 10 min' neonatal resuscitation before and after the use of the SDA. While on the mother's side, we obtained data on maternal outcomes following PPH management before and after the use of the SDA.

\section{Ethics approval}

This study has been approved by the Human Research Ethics Committee of the University of the Witwatersrand (M190258) and the University of Rwanda, College of Medicine and Health Sciences' Institutional Review Board (No.377/CMHS IRB/2018). Permission to collect data has been granted by the hospitals which have authorization of using aggregated patient data in research. Consents to track the SDA usage were obtained from nurses and midwives. This study was carried out in 
accordance with relevant guidelines and regulations in the Ethical Declarations.

\section{Statistical analysis}

The data were checked for errors and exported from Microsoft Excel (Microsoft Corporation) to Stata version 16 (StataCorp LLC) for cleaning and analysis. Descriptive summary statistics were computed on data including the hospital, age of the mother at childbirth, the weight of the newborn at birth, sex of the newborn, mode of delivery, the leading clinician during delivery, Apgar scores at 1, 5 and $10 \mathrm{~min}$ and the resuscitation action taken for the newborns. For the maternal outcome, descriptive statistics included the hospital, age of the mother at delivery, gestational age in weeks, Mode of delivery, the leading clinician during delivery, Blood loss in milliliters, causes of $\mathrm{PPH}$, the resuscitation action taken, mother outcome and cause of maternal death if dead. Further, the neonatal outcomes and maternal outcomes were compared at baseline and endline using Fisher's exact test. Significance level was set at $p<0.05$.

\section{Results}

Demographic characteristics of end-users of the SDA

In total, 54 healthcare providers, 21 nurses and 33 midwives used the SDA during the intervention period. More than a half of the participants were from Masaka district hospital (56\%), the majority of them were female (61\%), the participants had an average age of 33 years $(\mathrm{SD}=7.1)$. In terms of their level of education, most participants hold an advanced diploma (A1) in midwifery (50\%). Only one participant had the secondary school level (A2) in nursing. The majority of the nurses and midwives had less than 6 years of experience in the obstetric care (59\%), spent more than $10 \mathrm{~h}$ per week providing services in obstetric care $(74 \%)$, and participated in the care of more than ten deliveries per month (76\%). Four participants acknowledged having never used a smartphone before the study. Additional details are shown in Table 1.

\section{SDA usage tracking}

The SDA usage by nurses and midwives was tracked through the server of Maternity Foundation (Table 2). We found that the most frequently accessed feature was the action cards, which was used 199 times and 128 times in the midterm (at 3 months' intervention period) and endpoint (at 6 months' intervention period) respectively. The duration of time spent in the action cards by users varied from $282 \mathrm{~min}$ at midterm to $371 \mathrm{~min}$ at endpoint. The action cards were used by 66 users at mid-term and 80 users at endpoint. The number of users is higher than the study sample $(n=54)$ because some participants have downloaded the SDA on their own smartphones in
Table 1 The demographic characteristics of end-users of the SDA $(N=54)$

\begin{tabular}{|c|c|}
\hline & n (\%) \\
\hline \multicolumn{2}{|l|}{ Hospital Affiliation } \\
\hline Masaka District Hospital & $30(56)$ \\
\hline Nyamata District Hospital & $24(44)$ \\
\hline \multicolumn{2}{|l|}{ Sex } \\
\hline Male & $21(39)$ \\
\hline Female & $33(61)$ \\
\hline \multicolumn{2}{|l|}{ Level of Education } \\
\hline Midwife A0 & $6(11)$ \\
\hline Midwife A1 & $27(50)$ \\
\hline Nurse AO & $5(9)$ \\
\hline Nurse A1 & $15(28)$ \\
\hline Nurse A2 & $1(2)$ \\
\hline \multicolumn{2}{|c|}{ Experience in obstetrical care (years) } \\
\hline $1-5$ & $32(59)$ \\
\hline $6-10$ & $15(28)$ \\
\hline$>10$ & $7(13)$ \\
\hline \multicolumn{2}{|c|}{ Weekly workload in obstetric care (hours) } \\
\hline $0-5$ & $4(7)$ \\
\hline $6-10$ & $10(19)$ \\
\hline$>10$ & $40(74)$ \\
\hline \multicolumn{2}{|c|}{ Number of deliveries past month } \\
\hline $0-5$ & $8(15)$ \\
\hline $6-10$ & $5(9)$ \\
\hline$>10$ & $41(76)$ \\
\hline \multicolumn{2}{|c|}{ Experience with the smartphone } \\
\hline Tried using one & $50(93)$ \\
\hline Never tried using one & $4(7)$ \\
\hline Age, years, Mean (SD) & $33(7.1)$ \\
\hline
\end{tabular}

Abbreviations: \% Weighted percent, SD Standard deviation

addition to the phones provided by the researcher and they were using both phones at different occasions. The least accessed feature was the essential drugs, which was used 26 times and 87 times in the midterm and endpoint respectively. The duration of time spent in the essential drugs by users varied from $14 \mathrm{~min}$ at midterm to $33 \mathrm{~min}$ at endpoint. The essential drugs were consulted by 14 participants at midterm and 23 participants at endpoint. For some features (action cards and essential drugs) the number of participants using the feature and the times spent in the SDA increased from midterm to endpoint. While for other features (practical procedures and videos), the number of participants using the feature and the session counts decreased from midterm to endpoint.

With regards to 'MyLearning feature', Table 2 shows that a good portion of study participants $(n=44,85 \%)$ had started the familiar learning process in the midterm and by the endpoint $92 \%$ of the study participants had 
Table 2 SDA use by nurses and midwives

\begin{tabular}{|c|c|c|c|c|}
\hline \multirow[t]{3}{*}{ SDA App Features } & Midterm & & End-Point & \\
\hline & Session Count & Session duration & Session Count & Session duration \\
\hline & No. (number of times) & No. (duration in minutes) & No. (number of times) & No. (duration in minutes) \\
\hline Action Cards & $66(199)$ & $66(282)$ & $80(128)$ & $80(371)$ \\
\hline Essential drugs & $14(26)$ & $14(14)$ & $23(87)$ & $23(33)$ \\
\hline Practical Procedures & $37(87)$ & 37 (195) & $17(26)$ & $17(64)$ \\
\hline Videos & $53(104)$ & $53(50)$ & $33(62)$ & $32(47)$ \\
\hline My Learning & $\begin{array}{l}\text { Started the learning pro- } \\
\text { cess, No. (\%) }\end{array}$ & & $\begin{array}{l}\text { Passed the 'MyLearning' } \\
\text { surveys, No. (\%) }\end{array}$ & \\
\hline Familiar & $44(85)$ & & $48(92)$ & \\
\hline Proficient & $43(83)$ & & $40(77)$ & \\
\hline Expert & $39(75)$ & & $39(75)$ & \\
\hline SDA champions & & & $2(3.85)$ & \\
\hline $\begin{array}{l}\text { Global Total SDA down- } \\
\text { loads_by March 2020, } \\
\text { No. (\%) }\end{array}$ & $126,444(100)$ & & & \\
\hline $\begin{array}{l}\text { Total SDA downloads } \\
\text { in Rwanda_by March } \\
\text { 2020, No. (\%) }\end{array}$ & $179(0.14)$ & & & \\
\hline
\end{tabular}

Abbreviations: No Number of content users, \% Weighted percent

passed the familiar questionnaire. Similarly, a good portion of study participants $(n=43,83 \%)$ had started the proficient learning process in the midterm and by the endpoint $73 \%$ of the study participants had passed the proficient questionnaire. Finally, at endpoint, $75 \%$ of the study participants had passed the expert questionnaire. At the end of the intervention period, we had two SDA champions. The maternity foundation's server had also recorded 179 SDA users in Rwanda up to March 2020, meaning that our study participants in addition to downloading the SDA app on their own phones, they could have also shared the SDA to their colleagues in other hospitals apart from the hospitals included in the study. An exploration of the SDA acceptability was done at endline using surveys and focus group discussions with nurses and midwives. The findings of the SDA acceptability will be documented in a separate paper.

\section{Cases of newborns asphyxia}

The analysis included 126 cases of newborns asphyxia in the baseline and 87 in endline groups over the study period. Table 3 shows that the majority of cases were from Masaka district hospital in the baseline group (55\%) while in the endline group more cases were from Nyamata district hospital (56\%). There were more male newborns for the baseline group $(n=79,63 \%)$ and the endline group $(n=54,62 \%)$. The most frequent mode of delivery was the spontaneous vaginal delivery in both the baseline group $(n=77,61 \%)$ and the endline groups $(n=45,52 \%)$. The leading clinicians during delivery, also in charge of neonatal resuscitation were mainly midwives $(n=71,56 \%)$ in the baseline group and $(n=44,51 \%)$ in the endline group. The average age of the mother was similar, 28 years and 27 years in the baseline and endline groups, respectively. The average weight of the newborn was similar, $2900 \mathrm{~g}$, and $2800 \mathrm{~g}$ in the baseline and endline groups, respectively. The average Apgar score at birth was the same, $6 / 10$ in both the baseline and endline groups. However, the average Apgar score after $10 \mathrm{~min}$ of resuscitation was lower in the baseline group $(8 / 10)$ than the endline group (9/10). The resuscitation actions taken were mostly dominated by aspiration and ventilation with Ambu bag in both groups, baseline (82\%), and endline (79\%). The endline group had higher numbers of newborns with stable outcome after resuscitation $(n=64$, $74 \%)$ than the baseline group $(n=40,32 \%)$. Overall, the endline group had fewer newborns transferred to NICU or neonatology service $(n=24,28 \%)$ than the baseline group $(n=78,62 \%)$.

\section{Cases of postpartum haemorrhage (PPH)}

The analysis included 114 cases of Post-partum haemorrhage (PPH): 67 in baseline and 47 in endline groups for both Masaka and Nyamata district hospitals over the study period. Table 4 shows that the majority of cases were from Masaka district hospital in the baseline group (54\%) while in the endline group more cases were from Nyamata district hospital (55\%). The most frequent mode of delivery was the spontaneous vaginal delivery in both the baseline group $(n=47,70 \%)$ and the endline groups 
Table 3 Participants characteristics_Newborns asphyxia $(N=213)$

\begin{tabular}{|c|c|c|}
\hline & Baseline $\mathrm{n}(\%)$ & Endline $\mathrm{n}(\%)$ \\
\hline \multicolumn{3}{|l|}{ Hospital Affiliation } \\
\hline Masaka District Hospital & $69(55)$ & $38(44)$ \\
\hline Nyamata District Hospital & $57(45)$ & $49(56)$ \\
\hline \multicolumn{3}{|l|}{ Sex } \\
\hline Male & $79(63)$ & $54(62)$ \\
\hline Female & $47(37)$ & $38(38)$ \\
\hline \multicolumn{3}{|l|}{ Mode of Delivery } \\
\hline Caesarean Section & $49(39)$ & $42(48)$ \\
\hline Spontaneous Vaginal Delivery & $77(61)$ & $45(52)$ \\
\hline \multicolumn{3}{|l|}{ Leading Clinician of the Delivery } \\
\hline Doctor & $49(39)$ & $42(48)$ \\
\hline Midwife & $71(56)$ & $44(51)$ \\
\hline Nurse & $6(5)$ & $1(1)$ \\
\hline \multicolumn{3}{|l|}{ Resuscitation Actions } \\
\hline Aspiration, Ventilation with Ambu bag & $103(82)$ & $69(79)$ \\
\hline Aspiration, Ventilation, Oxgenotherapy & $12(9)$ & $6(7)$ \\
\hline CPR, Ventilation, Oxygenation & $11(9)$ & $12(14)$ \\
\hline \multicolumn{3}{|l|}{ Outcome after $10 \mathrm{~min}$ Resuscitation } \\
\hline Transferred to NICU or Neonatology Service & $78(62)$ & $24(28)$ \\
\hline Skin to skin bonding with mother & $39(31)$ & $63(72)$ \\
\hline Dead & $9(7)$ & $0(0)$ \\
\hline Weight of the newborn, grams, Median (IQR) & $2900(880)$ & $2800(800)$ \\
\hline Mother's age, years, Median (IQR) & $28(7)$ & $27(7)$ \\
\hline \multicolumn{3}{|l|}{ Apgar Scores, Median (IQR) } \\
\hline At $1 \mathrm{~min}$ & $6(2)$ & $6(2)$ \\
\hline At $5 \mathrm{~min}$ & $7(2)$ & $7(1)$ \\
\hline At $10 \mathrm{~min}$ & $8(2)$ & $9(2)$ \\
\hline
\end{tabular}

Abbreviations: \% Weighted percent, IQR Interquartile range

( $n=34,72 \%$ ). The leading clinicians of the delivery, also in charge of $\mathrm{PPH}$ management were mainly midwives $(n=36,54 \%)$ in the baseline group and $(n=25,53 \%)$ in the endline group. The average age of the mother was similar, 28years in the baseline and endline groups, respectively. The average gestational age was similar, 39 weeks in the baseline and endline groups, respectively. The mean estimated amount of blood loss was 1167.2 $\mathrm{mls}$ in the baseline group and $1178.7 \mathrm{mls}$ in the endline group. The most frequent cause of $\mathrm{PPH}$ was the uterine atony in both the baseline (72\%) and the endline group (70\%). The resuscitation actions taken were mostly dominated by administering oxytocin, IV fluids, and blood transfusion in both groups, baseline (37\%), and endline (55\%). The endline group had higher numbers of women with stable outcomes after $\mathrm{PPH}$ management $(n=44$, $94 \%)$ than the baseline group $(n=55,78 \%)$. Overall, the endline group had fewer women transferred to referral hospitals after PPH management $(n=3,6 \%)$ than the baseline group $(n=13,19 \%)$.

\section{Pre-post differences in newborns outcomes} following neonatal resuscitation

We found a statistically significant association between the SDA intervention and newborns' outcomes following neonatal resuscitation, 6 months after baseline (Table 5). Among 213 cases of newborns complications who were included in the study, stable outcomes following neonatal resuscitation were recorded in $31 \%$ of newborns cases at baseline and $72 \%$ at endline. The transferred (unstable) newborns were 62 and $28 \%$ at baseline and endline respectively. While death following neonatal resuscitation appeared in $7 \%$ for the baseline group only. No death was recorded in the endline group. There was a significant difference between baseline and endline in the outcome (stable, transferred, and dead) for newborns who underwent neonatal resuscitation ( $p=0.000$, Fisher's exact test).

\section{Pre-post differences in maternal outcomes following PPH management}

We found a statistically significant association between the SDA intervention and maternal' outcomes following 
Table 4 Participants characteristics_PPH $(N=114)$

\begin{tabular}{|c|c|c|}
\hline & Baseline n (\%) & Endline $\mathrm{n}(\%)$ \\
\hline \multicolumn{3}{|l|}{ Hospital Affiliation } \\
\hline Masaka District Hospital & $36(54)$ & $21(45)$ \\
\hline Nyamata District Hospital & $31(46)$ & $26(55)$ \\
\hline \multicolumn{3}{|l|}{ Mode of Delivery } \\
\hline Caesarean Section & $20(30)$ & $13(28)$ \\
\hline Spontaneous Vaginal Delivery & $47(70)$ & $34(72)$ \\
\hline \multicolumn{3}{|l|}{ Leading Clinicians of the Delivery } \\
\hline Doctor & $20(30)$ & $13(28)$ \\
\hline Midwife & $36(54)$ & $25(53)$ \\
\hline Nurse & $11(16)$ & $9(19)$ \\
\hline \multicolumn{3}{|l|}{ Causes of PPH } \\
\hline Uterine atony & $48(72)$ & $33(70)$ \\
\hline Cervical tear & $11(16)$ & $7(15)$ \\
\hline Retention of Placenta & $8(12)$ & $7(15)$ \\
\hline \multicolumn{3}{|l|}{ Resuscitation Actions } \\
\hline Oxytocin, IV fluids & $23(34)$ & $7(15)$ \\
\hline Oxytocin, IV fluids, Transfusion & $25(38)$ & $26(55)$ \\
\hline Repair of tear, IV fluids & $11(16)$ & $7(15)$ \\
\hline Removal of Placenta, IV fluids & $8(12)$ & $7(15)$ \\
\hline \multicolumn{3}{|c|}{ Maternal outcome after PPH Management } \\
\hline Transferred to Referral Hospital & $13(19)$ & $3(6)$ \\
\hline Stable & $52(78)$ & $44(94)$ \\
\hline Dead & $2(3)$ & $0(0)$ \\
\hline Mother's age, years, Median(IQR) & $28(5)$ & $28(5)$ \\
\hline Gestational age, weeks, Median(IQR) & $39(0)$ & $39(0)$ \\
\hline Blood loss in Mls, Mean (SD) & $1167.2(276.6)$ & $1178.7(257.1)$ \\
\hline
\end{tabular}

Abbreviations: \% Weighted percent, IQR Interquartile range, SD Standard deviation

PPH management, 6 months after baseline (Table 6). Among 114 cases of PPH who took part in the study, stable outcomes following PPH management were recorded in $78 \%$ PPH cases at baseline and $94 \%$ at endline. Transferred (unstable) women were 19 and 6\% at baseline and endline respectively. While death attributed to $\mathrm{PPH}$ appeared in $3 \%$ women for the baseline group only. No death was recorded in the endline group. There was a significant difference between baseline and endline in the maternal outcome (stable, transferred, and dead) following PPH management ( $p=0.048$, Fisher's exact test).

\section{Discussion}

\section{Main findings}

The current study investigated the use of the SDA and its relationship to $\mathrm{BEmONC}$ outcomes for the most frequent birth-related complications in Rwanda; PPH and newborn asphyxia. Apgar scores and PPH progressions were considered in the investigation of newborn and maternal outcomes following neonatal resuscitation and PPH management.

The findings from this study are consistent with SDA having the potential to improve clinical outcomes. These results are encouraging: nurses and midwives were willing to use SDA and the intervention was engaged with throughout the intervention period. Clinical data show a correlation between SDA implementation and improved maternal and newborn outcomes. However, the improved maternal and newborn outcomes may have been influenced by other factors in addition to the SDA intervention. Nevertheless, the findings of this study contribute to the broader knowledge about the SDA effectiveness and provide promising evidence to support the need for more rigorous and expensive research on the SDA.

\section{Comparison with other studies}

As was noted in the present study, Lund et al. [32] also reported a significant effect of the use of the SDA particularly on skilled birth attendants in terms of significantly increased knowledge and skills scores in neonatal resuscitation in Ethiopia [32]. Our previous study also found a significant association between the SDA uptake/use and change in knowledge and skills of nurses and midwives in PPH management and NR [33]. Another study conducted in DRC Congo to determine the feasibility, acceptability, and potential effect of the SDA on health workers' practices in BEmONC reported a significant increase of health worker knowledge and self-confidence in the management of obstetric and newborn emergencies after 3 months SDA intervention [37]. In addition to the previous studies on the SDA, the present study provided evidence on the SDA implementation, use, and clinical outcomes. However, the implicit theory of change for the SDA may follow a logical sequence of (a) the SDA implemented and used; (b) the use of SDA supports

Table 5 Newborns outcomes following neonatal resuscitation $(N=213)$

\begin{tabular}{llll}
\hline & Before SDA $(\boldsymbol{N}=\mathbf{1 2 6}), \mathbf{n}(\mathbf{W t} . \%)$ & After SDA (N=87), $\mathbf{n}(\mathbf{W t} . \%)$ & $P$-Value (Fisher' exact test) \\
\hline $\begin{array}{lll}\text { Newborns Outcome } \\
\text { Stable }\end{array}$ & $39(31)$ & $63(72)$ & $P($ Fisher $)=0.000$ \\
Unstable(Transferred) & $78(62)$ & $24(28)$ & \\
Dead & $9(7)$ & $0(0)$ & \\
\hline
\end{tabular}


Table 6 Maternal outcomes following PPH management $(N=114)$

\begin{tabular}{|c|c|c|c|}
\hline & Before SDA $(N=67), \mathrm{n}(\mathrm{Wt} . \%)$ & After SDA $(N=47), n(W t . \%)$ & $P$-Value (Fisher' exact test) \\
\hline \multicolumn{4}{|l|}{ Maternal outcome } \\
\hline Stable & $52(78)$ & $44(94)$ & $P($ Fisher $)=0.048$ \\
\hline Unstable(Transferred) & 13(19) & $3(6)$ & \\
\hline Dead & $2(3)$ & $0(0)$ & \\
\hline
\end{tabular}

Abbreviations: Wt.\% Weighted percent

skilled birth attendants' continuous learning and decision making; the use of the SDA changes clinical response behaviors and practices; (c) the changes in practices leads to improved clinical outcomes. Therefore, future research using a theory of change or a logical framework over a long period is needed to understand the dynamics and change processes of skilled birth attendants' clinical practices when using the SDA.

Moreover, mHealth programmes have shown promising results for newborn outcomes. A study which looked at antenatal messaging services using a mobile phone intervention in Zanzibar reported a significant reduction in perinatal mortality [38]. This could be explained by the fact that mhealth applications have the potentiality to put relevant and reliable healthcare information into the hands of healthcare workers thus helping them in the management of maternal and neonatal cases. But also, that health professionals need continuous access to updated healthcare information and clinical guidelines to support their decision making during practices. The same opinion was reported in a similar study done by Grol et al. [39] about the effective implementation of mHealth interventions [39]. However, Tamrat et al. [40] in their systematic review which analysed mHealth interventions in maternal and newborn health programs and their outcomes around the world reported that few trials have been conducted and most maternal mHealth programmes have not been evaluated, and the evidence is still weak [40]. Thus the need for more evidence on maternal and newborn mHealth programmes.

Maternal outcome following PPH management was another significant predictor in the present study. Our findings are in agreement with other studies that documented mHealth interventions which have been tested in a variety of health services delivery including decision support and clinical management [41-43]. These studies found promising findings for the mhealth technology in general and for maternal healthcare services in particular. Then again, other studies have shown that the evidence of maternal mHealth interventions is supportive but weak. A 2017 systematic review on mHealth interventions in low-income countries demonstrated good promise for the use of mHealth interventions in maternal health, but the evidence is limited [20]. This review suggested more research on the relationship between mHealth and clinical outcomes because the majority of mHealth trials focused on very distinct mhealth applications (i.e appointment reminders and data collection). Another review which identified $51 \mathrm{RCTs}$, documented that nearly half of the reviewed RCTs $(n=22)$ showed negative or unclear results, thus the need for better evidence; and for caution with new interventions [44]. Another study which assessed the impact of the SDA on the incidence of PPH in Ghana reported that the SDA was associated with an insignificant lower incidence of PPH [45]. Hence, the need for more evidence and higher-quality research in this field. The current study adds to much-needed evidence as to how a decision-support mHealth application functioned in a low-resource setting. Future research that analyze event-event correlation between SDA use and maternal and newborn outcomes would be important.

\section{Limitations}

Though our study documents important patterns of use of a mHealth to inform clinical decisions, the quantity of information accessed for the care of individual patients is limited in the current study. We acknowledge the limitations of our study design, pre-post intervention, which partly permitted us to demonstrate the effects of the SDA on maternal and newborn outcomes. The observed maternal and newborn outcomes may have been influenced by other factors in addition to the SDA intervention. Also, nurses and midwives may have consulted other sources rather than the SDA to inform their decision-making during the intervention. Although this limitation is inherent in the design of the study, the current study contributes to the broader evidence about the effectiveness of the SDA and provides much-needed insights as to how a decision-support mHealth application functioned in a low-resource setting.

\section{Conclusion}

The use of the SDA supported nurses and midwives in the management of $\mathrm{PPH}$ and neonatal resuscitation which may have contributed to improved maternal and neonatal outcomes during 6 months of the SDA intervention. Mhealth interventions that focus on the clinical decision support process in maternal and newborn care 
may contribute to improved services delivery and should be considered by policymakers in resource-limited settings, like Rwanda. Overall, there is limited evidence on the effects of mHealth interventions on clinical outcomes on a large scale and further research is necessary to draw holistic conclusions, particularly for developing countries within the field of maternal and newborn care.

\begin{abstract}
Abbreviations
BEmONC: Basic Emergency Obstetric and Newborn Care; CHWs: Community health workers; CMHS IRB: College of Medicine and Health Sciences Institutional Review Board; IQR: Interquartile range; IV: Intravenous line; LMICs: Low- and middle-income countries; mHealth: Mobile Health; Mls: Millilitres; NICU: Neonatal intensive care unit; SDA: Safe Delivery mhealth Application; SD: Standard deviation; PPH: Post-Partum Hemorrhage; NR: Neonatal Resuscitation; SBA: Skilled Birth Attendants; WHO: World Health Organisation.
\end{abstract}

\section{Acknowledgments}

We would like to thank The Ministry of Health, Rwanda, and The Maternity Foundation for supporting this research. We also acknowledge the Consortium for Advanced Research and Training (CARTA) for funding this research.

\section{Authors' contributions}

$\mathrm{AN}, \mathrm{LI}, \mathrm{MN}$, and DNC were involved in the conception and design of the study. AN drafted the manuscript. LI, MN, and DNC reviewed the manuscript. All authors read and approved the final manuscript.

\section{Funding}

This research was supported by the Consortium for Advanced Research Training in Africa (CARTA). CARTA is jointly led by the African Population and Health Research Center and the University of the Witwatersrand and funded by the Carnegie Corporation of New York (Grant No--B 8606.R02), Sida (Grant No:54100113), the DELTAS Africa Initiative (Grant No: 107768/Z/15/Z) and Deutscher Akademischer Austauschdienst (DAAD). The DELTAS Africa Initiative is an independent funding scheme of the African Academy of Sciences (AAS)'s Alliance for Accelerating Excellence in Science in Africa (AESA) and supported by the New Partnership for Africa's Development Planning and Coordinating Agency (NEPAD Agency) with funding from the Wellcome Trust (UK) and the UK government. The statements made and views expressed are solely the responsibility of the Fellow". The funding body/bodies played no role in the design of the study and data collection process, analysis, interpretation of data, and in writing the manuscript.

\section{Availability of data and materials}

The dataset generated for this study will be made available from the corresponding author on a reasonable request.

\section{Declarations}

\section{Ethics approval and consent to participate}

This study has been approved by the Human Research Ethics Committee of the University of the Witwatersrand (M190258) and the University of Rwanda, College of Medicine and Health Sciences'Institutional Review Board (No.377/ CMHS IRB/2018). Permission to collect data has been granted by the hospitals.

\section{Consent for publication}

Not applicable.

\section{Competing interests}

None declared.

\section{Author details}

'School of Public Health, Faculty of Health Sciences, University of the Witwatersrand, 1 Smuts Avenue, Braamfontein, Johannesburg 2000, South Africa. ${ }^{2}$ School of Public Health / College of Medicine and Health Sciences, University of Rwanda, P.O. Box 3286, Kigali, Rwanda. ${ }^{3}$ Nigerian Institute of Medical
Research, 6 Edmund Cres, Yaba, Lagos, Nigeria. ${ }^{4}$ Department of Biomedical Statistics and Informatics, Vrije Universiteit Brussel, Brussels, Belgium.

Received: 23 March 2021 Accepted: 11 January 2022

Published online: 20 January 2022

\section{References}

1. Payne JD. The state of standards and interoperability for mHealth among low-and middle-income countries. 2013. http://www.mhealthkno wledge.org/sites/default/files/12_state_of_standards_report_2013.pdf. Accessed 28 Mar 2018.

2. Free $C$, Phillips $G$, Watson $L$, et al. The effectiveness of mobile-health technologies to improve health care service delivery processes: a systematic review and meta-analysis. PLoS Med. 2013;10(1):e1001363. https://doi. org/10.1371/journal.pmed.1001363 Epub ahead of print.

3. Hall CS, Fottrell E, Wilkinson S, et al. Assessing the impact of mHealth interventions in low- and middle-income countries - what has been shown to work? Glob Health Action. 2014;7:25606. https://doi.org/10. 3402/gha.v7.25606 Epub ahead of print.

4. Lee SH, Nurmatov UB, Nwaru Bl, et al. Effectiveness of mHealth interventions for maternal, newborn and child health in low- and middle-income countries: systematic review and meta-analysis. J Glob Health. 2016;6. https://doi.org/10.7189/jogh.06.010401 Epub ahead of print.

5. Wallis $L$, Blessing $P$, Dalwai $M$, et al. Integrating mHealth at point of care in low- and middle-income settings: the system perspective. Glob Health Action. 2017;10. https://doi.org/10.1080/16549716.2017.1327686 Epub ahead of print.

6. Tomlinson M, Rotheram-Borus MJ, Swartz L, et al. Scaling up mHealth: where is the evidence? PLoS Med. 2013;10:e1001382.

7. Huq NL, Azmi AJ, Quaiyum MA, et al. Toll free mobile communication: overcoming barriers in maternal and neonatal emergencies in rural Bangladesh. Reprod Health. 2014;11:1-12.

8. Martinez B, Ixen EC, Hall-Clifford $\mathrm{R}$, et al. mHealth intervention to improve the continuum of maternal and perinatal care in rural Guatemala: a pragmatic, randomized controlled feasibility trial. Reprod Health. 2018;15:120.

9. Ilozumba O, Abejirinde IOO, Dieleman M, et al. Targeting strategies of mHealth interventions for maternal health in low and middle-income countries: a systematic review protocol. BMJ Open. 2018;8. https://doi. org/10.1136/bmjopen-2017-019345 Epub ahead of print.

10. Mburu S, Oboko R. A model for predicting utilization of mHealth interventions in low-resource settings: case of maternal and newborn care in Kenya. BMC Med Inform Decis Mak. 2018;18. https://doi.org/10.1186/ s12911-018-0649-z.

11. Amoakoh HB, Klipstein-Grobusch K, Amoakoh-Coleman M, et al. The effect of a clinical decision-making mHealth support system on maternal and neonatal mortality and morbidity in Ghana: study protocol for a cluster randomized controlled trial. Trials. 2017;18:157.

12. Philbrick WC. mHealth and MNCH: state of the evidence. Trends, gaps, stakeholder needs, and opportunities for future research on the use of mobile technology to improve maternal, newborn, and child health. mHealth Alliance; 2013. http://www.healthenabled.org/resources/ pmnch_mhealth_evidence.pdf. Accessed 26 May 2017.

13. Nurmatov UB, Lee $\mathrm{SH}, \mathrm{Nwaru} \mathrm{Bl}$, et al. The effectiveness of mHealth interventions for maternal, newborn and child health in low- and middleincome countries: protocol for a systematic review and meta-analysis. J Glob Health. 2014;4:010407.

14. Hagar C, Kartzinel H. Healthcare information for all by 2015: preliminary findings and future direction. Inf Dev. 2016;32:354-61.

15. Thomsen CF, Barrie AMF, Boas IM, et al. Health workers' experiences with the Safe Delivery App in West Wollega Zone, Ethiopia: a qualitative study. Reprod Health. 2019;16:50.

16. Granja C, Janssen W, Johansen MA. Factors determining the success and failure of ehealth interventions: systematic review of the literature. J Med Internet Res. 2018;20. https://doi.org/10.2196/10235 Epub ahead of print.

17. Labrique $A B$, Vasudevan $L$, Kochi $E$, et al. mHealth innovations as health system strengthening tools: 12 common applications and a visual framework. 2015. http://southasiainstitute.harvard.edu/website/wp-content/ uploads/2014/08/MHEALTHINNOVATIONS.pdf. Accessed 20 Mar 2017. 
18. Agapitova N, Navarrete Moreno C. Dimagi: improving maternal and newborn care: World Bank; 2017. https://elibrary.worldbank.org/doi/abs/ $10.1596 / 27661$

19. Amoakoh HB, Klipstein-Grobusch K, Agyepong IA, et al. Can an mhealth clinical decision-making support system improve adherence to neonatal healthcare protocols in a low-resource setting? BMC Pediatr. 2020;20:1-13.

20. Colaci D, Chaudhri S, Vasan A. mHealth interventions in low-income countries to address maternal health: a systematic review. Ann Glob Health. 2017;82:922

21. Sondaal SFV, Browne JL, Amoakoh-Coleman M, et al. Assessing the effect of mHealth interventions in improving maternal and neonatal care in low- and middle-income countries: a systematic review. PLoS One. 2016;11. https://doi.org/10.1371/journal.pone.0154664 Epub ahead of print.

22. Adepoju I-OO, Albersen BJA, De Brouwere $V$, et al. $m$ Health for clinical decision-making in sub-Saharan Africa: a scoping review. JMIR mHealth uHealth. 2017;5:e38

23. Maternity Foundation M for mothers. What is the SDA app - Maternity Foundation. 2014. https://www.maternity.dk/about-the-app/what-is-theapp/. Accessed 26 July 2018.

24. Rwanda NI of S of, Planning/Rwanda M of F and E, Health/Rwanda M of et al. Rwanda demographic and health survey 2014-15. 2016. https:// dhsprogram.com/publications/publication-fr316-dhs-final-reports.cfm. Accessed 14 Nov 2019.

25. Sayinzoga F, Bijlmakers L, van der Velden K, et al. Severe maternal outcomes and quality of care at district hospitals in Rwanda-a multicentre prospective case-control study. BMC Pregnancy Childbirth. 2017;17:394.

26. MOH RHP. Republic of Rwanda. 2015. http://www.moh.gov.rw/fileadmin/ templates/policies/Health_Sector_Policy__19th_January_2015.pdf. Accessed 7 Aug 2017.

27. Wilmot $E$, Yotebieng M, Norris A, et al. Missed opportunities in neonatal deaths in Rwanda: applying the three delays model in a cross-sectional analysis of neonatal death. Matern Child Health J. 2017;21:1121-9.

28. Sayinzoga F, Bijlmakers L, Van Dillen J, et al. Maternal death audit in Rwanda 2009-2013: a nationwide facility-based retrospective cohort study. BMJ Open. 2016:6:9734

29. Kasine $Y$, Babenko-Mould Y, Regan S. Translating continuing professional development education to nursing practice in Rwanda: enhancing maternal and newborn health. Int J Africa Nurs Sci. 2018;8:75-81.

30. Puri R, Rulisa S, Joharifard S, et al. Knowledge, attitudes, and practices in safe motherhood care among obstetric providers in Bugesera, Rwanda. Int J Gynecol Obstet. 2012;116:124-7.

31. Nishimwe A, Conco DN, Nyssen M, et al. Context specific realities and experiences of nurses and midwives in basic emergency obstetric and newborn care services in two district hospitals in Rwanda: a qualitative study. BMC Nurs. 2022;21:9.

32. Lund S, Boas IM, Bedesa T, et al. Association between the safe delivery app and quality of care and perinatal survival in Ethiopia: a randomized clinical trial. JAMA Pediatr. 2016;170:765-71.

33. Nishimwe A, Ibisomi L, Nyssen $M$, et al. The effect of an mLearning application on nurses' and midwives' knowledge and skills for the management of postpartum hemorrhage and neonatal resuscitation: pre-post intervention study. Hum Resour Health. 2021;19:1-10.

34. Nishimwe A, Nyssen M, Ibisomi L, Nozizwe Conco D. Clinical decision making in basic emergency obstetric and newborn care among nurses and midwives: the role of the safe delivery mhealth application_prepost intervention study (research protocol). Inform Health Soc Care. 2021;46(2):126-35.

35. Namusoke $H$, Nannyonga MM, Ssebunya R, et al. Incidence and short term outcomes of neonates with hypoxic ischemic encephalopathy in a Peri Urban teaching hospital, Uganda: a prospective cohort study. Matern Health Neonatol Perinatol. 2018;4:6.

36. Website MF. Our Work - Maternity Foundation. https://www.maternity.dk/ our-work/. Accessed 23 July 2020.

37. Bolan NE, Sthreshley L, Ngoy B, et al. mLearning in the Democratic Republic of the Congo: a mixed-methods feasibility and pilot cluster randomized trial using the safe delivery app. Glob Health Sci Pract. 2018;6:693-710.
38. Lund $S$, Rasch $V$, Hemed $M$, et al. Mobile phone intervention reduces perinatal mortality in zanzibar: secondary outcomes of a cluster randomized controlled trial. JMIR mHealth uHealth. 2014:2:e15.

39. Grol R, Grimshaw J. From best evidence to best practice: effective implementation of change in patients' care. Lancet. 2003;362:1225-30.

40. Tamrat T, Kachnowski S. Special delivery: an analysis of mHealth in maternal and newborn health programs and their outcomes around the world. Matern Child Health J. 2012;16:1092-101.

41. McNabb M, Chukwu E, Ojo O, et al. Assessment of the quality of antenatal care services provided by health workers using a mobile phone decision support application in northern Nigeria: a pre/post-intervention study. PLoS One. 2015;10. https://doi.org/10.1371/journal.pone.0123940 Epub ahead of print.

42. Ngoc NTN, Bracken H, Blum J, et al. Acceptability and feasibility of phone follow-up after early medical abortion in Vietnam: a randomized controlled trial. Obstet Gynecol. 2014;123:88-95.

43. Chib A. The Aceh Besar midwives with mobile phones project: design and evaluation perspectives using the information and communication technologies for healthcare development model. J Comput Commun. 2010;15:500-25.

44. Chen $\mathrm{H}$, Chai Y, Dong L, et al. Effectiveness and appropriateness of mHealth interventions for maternal and child health: systematic review. JMIR mHealth uHealth. 2018;6:e7.

45. Klokkenga CMB, Enemark U, Adanu R, et al. The effect of smartphone training of Ghanaian midwives by the Safe Delivery application on the incidence of postpartum hemorrhage: a cluster randomised controlled trial. Cogent Med. 2019;6:1632016.

\section{Publisher's Note}

Springer Nature remains neutral with regard to jurisdictional claims in published maps and institutional affiliations.

Ready to submit your research? Choose BMC and benefit from

- fast, convenient online submission

- thorough peer review by experienced researchers in your field

- rapid publication on acceptance

- support for research data, including large and complex data types

- gold Open Access which fosters wider collaboration and increased citations

- maximum visibility for your research: over $100 \mathrm{M}$ website views per year

At BMC, research is always in progress.

Learn more biomedcentral.com/submissions 